\title{
Decipherment of Latent Handwritten Impressions - A Case Study
}

\author{
Ankit Srivastava $^{1 *}$, Meenakshi Mahajan ${ }^{2}$ and Kriti Nigam \\ ${ }^{1}$ Institute of Forensic Science \& Criminology, Bundelkhand University, Jhansi, U.P, INDIA \\ ${ }^{2}$ Regional Forensic Science Laboratory, Dharamshala, Himachal Pradesh, India
}

\begin{abstract}
The present work is based on a case received in the State Forensic Science Laboratory, Junga, Himachal Pradesh, India. The laboratory was asked to fix the identity of the deceased by examining the latent impressions present on an identity card found at the crime scene, near the corpse. The writing on the identity card was completely washed out due to weathering, leaving behind only some latent impressions made by the writing instrument in place of writing. The laboratory successfully deciphered the identity of deceased by carefully examining these latent impressions even after a time lapse of about a year. A number of techniques were applied but stereomicroscope again emerges out as a versatile tool for surface examination and gave the best results.
\end{abstract}

Keywords: Latent Impressions; ESDA; VSC; Stereomicroscope

\section{Introduction}

A number of questioned document cases are reported in the Document Division of a Forensic Science Laboratory, each is unique in its own way. Forensic document examiners use their expertise to evaluate documents. The scope of a document examiner's duties extends far beyond mere authentication of writings and signatures. Forensic document examiners analyse all aspects of the document; examination and decipherment of content from indentations and other latent impressions is just one of them.

Huber, defined handwriting as a complex motor skill which is a combination of sensory, neurological and physiological impulses. Factors such as visual perception and acuity, comprehension of form, central nervous system pathways and the anatomy and physiology of the bones and muscles of the hand and arm all combine to produce the desired [1]. In order to implement this acquired neuromuscular skill, two mandatory requirements are- a writing instrument i.e. pen, pencil, etc and a writing surface. Paper is the most common writing surface. It is a thin material usually produced by pressing together moist fibres of cellulose pulp derived from wood, rags or grasses, and drying them into flexible sheets [2].

Whenever writing is implemented on any paper with the help of any writing instrument, these fibres get disturbed due to the pressure implemented by the writing instrument, leaving behind depressions in the paper which are usually filled up by the ink provided by that wring instrument. Ink present on paper is prone to fading due to various unfavourable environmental conditions. In such conditions, these latent impressions could still prove to be useful in deciphering the content of document.

\section{Case History}

In the present case, a partially mummified dead body was found in forest area of District Solan, Himachal Pradesh, India. The body was mutilated and decomposed to such an extent that it could not be identified. On the basis of personal belongings, the deceased was positively identified by his family members, whose missing complaint was filed by his family around six months ago. After autopsy, the body was handed over to the family members and was finally cremated. Case seemed to be a little sorted at this point as the question related to the identity of corpse was successfully settled now.

Six months later, the wife of deceased received an anonymous call that her husband is still alive. On the basis of this phone call, wife of deceased lodged a complaint in Hon'ble High Court of Himachal Pradesh as to the confirmation of identity of deceased. High Court referred this case to Forensic Science Laboratory, Himachal Pradesh for ascertaining the identity of deceased (Figures 1A-1D).

Laboratory go through all the details of the case from its very inception and found out that in addition to various personal articles like ring, watch, etc., the belongings of the deceased also had an Identity Card, which was found at the crime scene, near the corpse bearing the printed matter titled "Himachal Pradesh Public Works Department" and various heads namely, Name, Designation \& Date of Issue. However, the handwritten portion under all these heads was completely washed / wiped off due to the moist environmental conditions as shown in (Figure 2A and 2B) mentioned below. Since the body was already
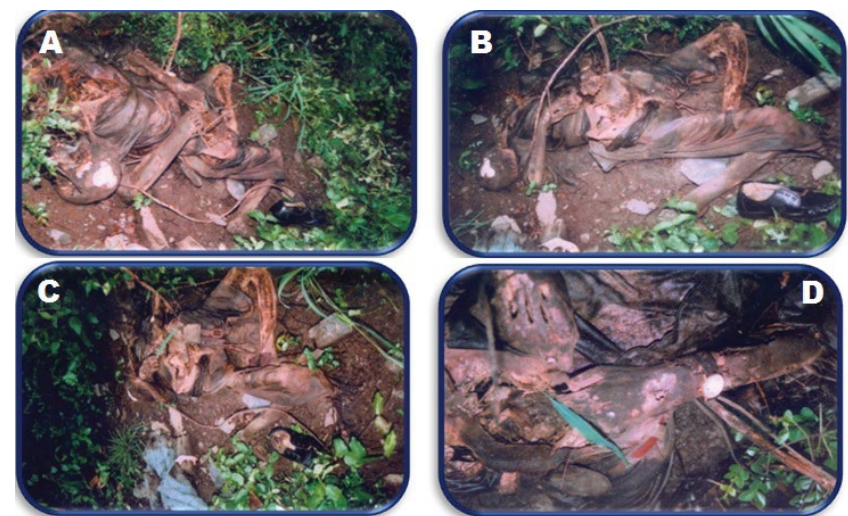

Figure 1: Partially mummified body found in forests of district solan.

${ }^{*}$ Corresponding author: Ankit Srivastava, Assistant Professor Institute of Forensic Science \& Criminology,Bundelkhand University, Jhansi, U.P, India, Tel: + 91-9415067667 E-mail: ankit_forensic81@rediffmail.com

Received September 29, 2017; Accepted October 09, 2017; Published October 15,2017

Citation: Srivastava A, Mahajan M, Nigam K (2017) Decipherment of Latent Handwritten Impressions - A Case Study. J Forensic Res 8: 392. doi: 10.4172/21577145.1000392

Copyright: (c) 2017 Srivastava A, et al. This is an open-access article distributed under the terms of the Creative Commons Attribution License, which permits unrestricted use, distribution, and reproduction in any medium, provided the original author and source are credited. 
Citation: Srivastava A, Mahajan M, Nigam K (2017) Decipherment of Latent Handwritten Impressions - A Case Study. J Forensic Res 8: 392. doi: 10.4172/2157-7145.1000392

Page 2 of 2
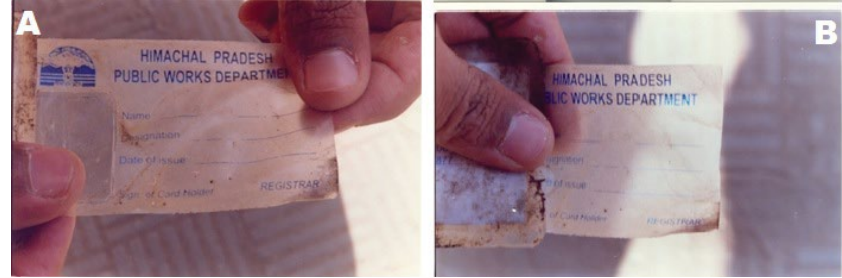

Figure 2: Identity card found near the mummified body.

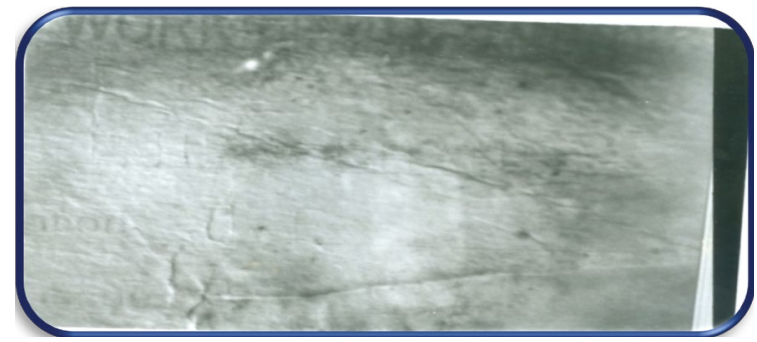

Figure 3: VSC image of ID Card.
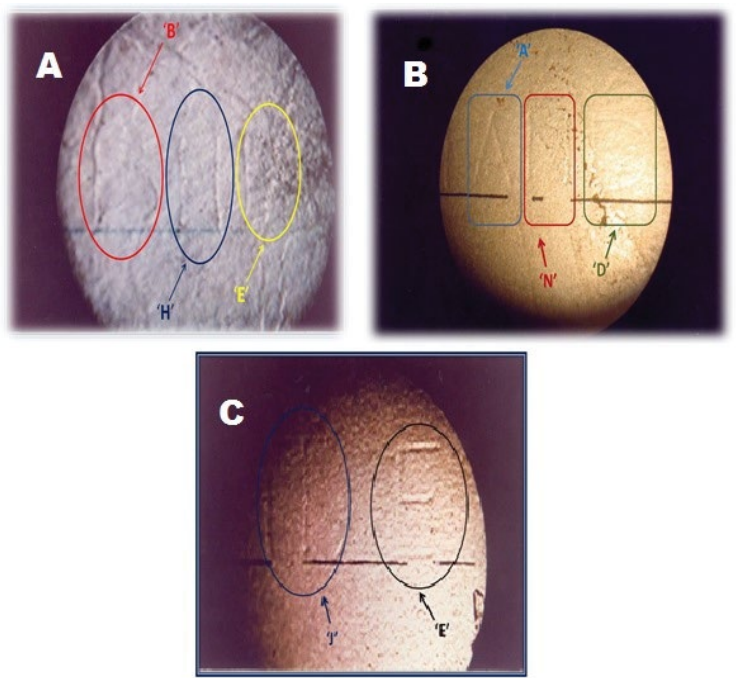

Figure 4: A: Deciphered letters- ' $B$ ', ' $H$ ' and ' $E$ '; $B$ : Deciphered letters- ' $A$ ', 'N' and 'D'; C: Deciphered letters- 'J' and 'E'.

cremated, no alternate way was left out to ascertain the identity of the deceased except from decipherment of latent impressions present on the identity card, which was the biggest challenge faced by State Forensic Science Laboratory, Junga in the present case.
Following systematic procedure had been adopted in deciphering handwritten latent impressions:

\section{Photography}

The identity card was firstly taken out of the plastic cover with utmost care and photographed under high resolution Nikon camera.

\section{Instrumental methods}

Firstly, the ID card was carefully examined under ESDA, but it failed to decipher the content in this case. After this, it was attempted to decipher under VSC-2000, the results were still inconclusive as shown in (Figure 3).

Finally attempts had been made using Getner- Stereomicroscope which gave the results as mentioned below. All the deciphered letters and numerals were carefully assembled to decipher the name and designation mentioned on the ID card.

\section{Result and Discussion}

The latent impressions on the identity card revealed out the name of deceased to be "BHEEM CHAND" and Designation as "J.E." (Figures 4A-4C). Date of issue was however, could not be clearly deciphered from the card. Next complication that arose in this case was that the F.I.R. spelled the name of deceased as "BHIM CHAND" which didn't match with the one deciphered from the ID card. The data was then matched with the service record of the deceased, which confirmed his name as "BHEEM CHAND".

\section{Conclusion}

A number of computational tools have been developed that not only helps in image processing but also in identification and verification of handwriting [3]. Along with this several sophisticated instruments are there which are able to make in depth analysis of document, still stereomicroscope once again proved itself to be a valuable tool in conducting the preliminary surface examination of various physical evidences and has proved its significance in Document Examination as well, by deciphering the latent impressions in the present case.

\section{Acknowledgement}

The authors are thankful to the Director, Forensic Science Laboratory, Himachal Pradesh for his inspiration \& support in presenting this case study in research form. The authors are also thankful to staff members of Document/ Photo Division for the help rendered in examination of the case.

\section{References}

1. Huber RA, Headrick AM (1999) Handwriting identification: Facts and fundamentals. CRC Press-Taylor \& Francis, Boca Raton, Florida.

2. Hilton O (1982) Scientific examination of questioned documents. Elsevier. New York.

3. Nigam K, Saran V, Gupta AK, Ahmed S (2013) Emerging trends of automated handwriting analysis. International Research Journal of Management Science \& Technology 4: 13-25
Citation: Srivastava A, Mahajan M, Nigam K (2017) Decipherment of Latent Handwritten Impressions - A Case Study. J Forensic Res 8: 392. doi: 10.4172/2157-7145.1000392 